\title{
State selective measurements of HCI produced by strong ultrashort laser-clusters interaction
}

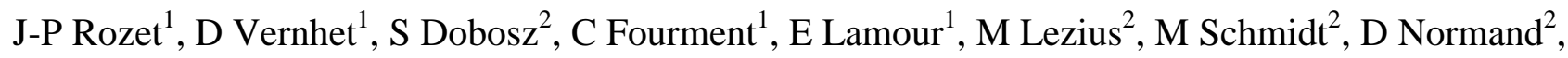

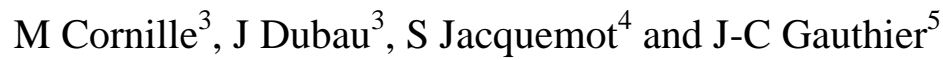 \\ ${ }^{1}$ Groupe de Physique des Solides, CNRS UMR 75-88, Universités Paris 7 et Paris 6, 75251 Paris Cedex 05, France \\ ${ }^{2}$ CEA-Saclay, DSM/DRECAM/SPAM Bât. 522, 91191 Gif-sur-Yvette Cedex, France \\ ${ }^{3}$ DARC, Observatoire de Paris, Place Jules Janssen, 92195 Meudon Cedex, France \\ ${ }^{4}$ CEA-DAM/Ile de France, BP12, 91680 Bruyeres le Chatel, France \\ ${ }^{5}$ LULI, CNRS UMR 76-05, Ecole Polytechnique, 91128 Palaiseau Cedex, France
}

PACS Ref: $52.50 . J m, 34.80 . \mathrm{Kw}, 36.40 . \mathrm{c}, 52.25 . \mathrm{Nr}$

\begin{abstract}
We have performed studies of keV x-ray production from $(\mathrm{Ar})_{\mathrm{n}},(\mathrm{Kr})_{\mathrm{n}}$ and $(\mathrm{Xe})_{\mathrm{n}}$ rare gas clusters (with $\mathrm{n}$ between $10^{4}$ and $10^{6}$ atoms/cluster) submitted to intense $\left(\leq 10^{18} \mathrm{~W} / \mathrm{cm}^{2}\right)$ infrared $(790 \mathrm{~nm})$ laser pulses. We have determined the photon energies and the absolute photon emission yields as a function of several physical parameters governing the interaction: size and atomic number of the clusters, peak intensity of the laser. Up to $10^{6} 3 \mathrm{keV}$ photons per pulse at a moderate $\left(10^{15} / \mathrm{cm}^{2}\right)$ atomic density have been observed. High resolution spectroscopy studies in the case of $(\mathrm{Ar})_{\mathrm{n}}$ clusters have also been performed, giving unambiguous evidence of highly charged (up to heliumlike) ions with $\mathrm{K}$ vacancies production. The results obtained indicate that $\mathrm{x}$-rays are emitted before cluster explosion on a subpicosecond time scale, and shed some light on the mechanisms involved in the first stage of the production of the nanoplasma induced from each cluster.
\end{abstract}

\section{Introduction}

Table-top devices are now available that can easily produce subpicosecond laser pulses with intensities easily exceeding $10^{18} \mathrm{~W} / \mathrm{cm}^{-2}$. This has open a new area in the field of laser-matter interaction, including the generation of high order harmonic in the UV range, high charge states and $\mathrm{keV} \mathrm{X}$-rays using clusters or solids. A major subject of interest is the possibility of constructing cheap versatile sources producing intense and/or short X-ray pulses for applications. Cluster targets have received considerable attention in recent years for several reasons. In the first place, they fill the gap between solid and gaseous targets, they offer the opportunity of observing well isolated nanoplasmas, and constitute simple objects where the laser-matter interaction may be studied more easily. Second, they constitute renewable targets free of debris and can nevertheless be very efficient in absorbing laser energy, and may then be a very convenient tool to produce intense X-ray and fast electron as well as highly charged ions emission. Hot electrons with energies of a few $\mathrm{keV}$ [1] and multicharged ions with energies reaching the $\mathrm{MeV}$ have been observed [2-4]. Up to now, X-ray emission studies have been mainly limited to qualitative observations and theoretical discussions [5-11], but a critical analysis of the predictions of various models which have been proposed in terms of experimental observables is still lacking. We are conducting an experimental research program which is an effort to measure absolute X-ray emission yields and ionic charge state distributions as a function of various physical parameters governing the laser-cluster interaction : these parameters include the cluster size, atomic number and total atomic density on one hand, and the laser pulse energy, pulse duration, wavelength and polarisation on the other hand. We present here some of the results obtained so far and discuss them in terms of possible mechanisms for the production of highly charged ions.

\section{Experimental setup}

The experimental set-up, including the apparatus used for cluster generation and the X-ray spectrometers, has been described in detail in previous publications $[3,8,12]$. Briefly, clusters are generated within a pulsed adiabatic expansion of the well documented Hagena type [13]. This device leads to a mean cluster $\tilde{N}_{c}$ size given by

$$
\tilde{N}_{c} \approx A_{c} P_{0}^{1.95},
$$

where $\mathrm{P}_{0}$ is the backing pressure (bar) and $\mathrm{A}_{\mathrm{c}}$ is a quantity depending on the atomic species, the temperature and the geometry of the nozzle. In our case, $A_{c}$ amounts to $2.110^{3}, 6.710^{3}$ and $2.310^{4}$ 
respectively for $\mathrm{Ar}, \mathrm{Kr}$ and $\mathrm{Xe}$ species. The atomic beam density $\rho_{c}$ at a working distance from the nozzle of $475 \mathrm{~mm}$ follows a linear dependence on the backing pressure given by

$$
\rho_{\mathrm{c}} \approx 5 \times 10^{13} \mathrm{P}_{0} \mathrm{~cm}^{-3} \text {. }
$$

The intense laser field is generated with a Ti:sapphire laser system delivering pulses of minimum duration of $70 \mathrm{fs}$ at 790 or $395 \mathrm{~nm}$ with a repetition rate of $20 \mathrm{~Hz}$. The beam diameter is approximately $45 \mathrm{~mm}$ and the maximum pulse energy available in the interaction zone is $83.5 \mathrm{~mJ}$. The laser light is focused with a $\mathrm{f}=170 \mathrm{~mm}$ off-axis parabolic mirror leading to a maximum peak intensities $I_{\text {peak }}^{\max } \sim 10^{18} \mathrm{Wcm}^{-2}$. The $\mathrm{I}_{\text {peak }}$ values are determined from the saturation intensities in the optical field ionisation (OFI) of a low density atomic Neon target using the barrier suppression ionisation model (BSI) [14]. The precision of this method is estimated to be $\pm 20 \%$.
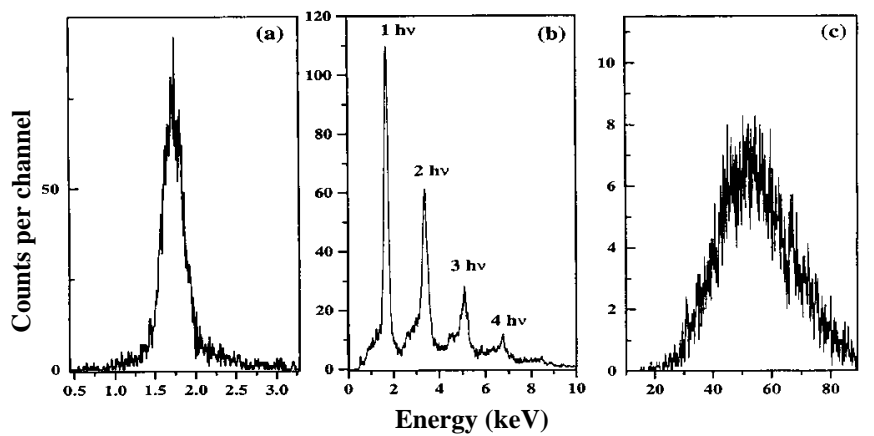

Fig. 1. X-ray spectra obtained after irradiation of krypton clusters. Spectrum (a) is recorded at very low count rate by using a diaphragm in front of the detector, spectrum (b) correspond to about one detected photon per laser pulse and spectrum (c) to a large count rate (about 30 detected photons per pules).

The X-rays are analysed using two $\mathrm{Si}(\mathrm{Li})$ detectors and crystal spectrometer. The $\mathrm{Si}(\mathrm{Li})$ detectors are sealed with thin $(25 \mu \mathrm{m})$ Be windows and can be positioned to different angles $\left(0^{\circ}, 100^{\circ}\right.$ and $\left.145^{\circ}\right)$ with respect to the laser beam. If a reduction in the counting rates of these very efficient detector systems is desired, their typical solid angle of detection of $\sim 5 \times 10^{-3} \mathrm{sr}$ can be reduced with the help of small aperture diaphragms and/or thin aluminum-coated Mylar foils. For low count rates, a single peak is observed at the characteristic energy of the observed atomic transition ("one-photon spectrum"); for higher count rates, several photons are detected within one laser shot, and pileup occurs: peaks at two, three, four... times the position of the single photon peak are observed, obeying a Poissonian distribution; finally, for very high count rates, a Gaussian peak is observed, whose position is proportional to both the photon energy and the simultaneously detected number of photons. This effect is illustrated in figure 1 in the case when the observed transition is the $1.75 \mathrm{keV}$ $\mathrm{X}_{\mathrm{L}}$ line of $\mathrm{Kr}$.

State resolved measurements are performed using a high-resolution high-transmission Bragg-crystal spectrometer equipped with a flat mosaic graphite crystal (HOPG) and a position sensitive detector working in the photon counting mode. Typical integrated efficiency and resolving power of this spectrometer are respectively $210^{-6}$ and 2000.

\section{Results}

We first present the absolute photon yields measured with the $\mathrm{Si}(\mathrm{Li})$ detectors for $\mathrm{Ar}, \mathrm{Kr}$ and Xe clusters. More detailed information obtained with the crystal spectrometer are presented in the next subsection.

\subsection{Low resolution results}

Systematic studies of the emission pattern have been performed using three different arrangements of the positions of the two $\mathrm{Si}(\mathrm{Li})$ detectors [8]. In all cases, an isotropic emission pattern is observed within the experimental error of $\pm 5 \%$. Similarly, no dependence on the orientation of the polarisation vector of the laser light has been found. The absolute photon yield can then been extracted from the total counts observed in any of our detectors of known detection efficiency and the simultaneously recorded number of laser pulses. Typical one-photon spectra corresponding to $\mathrm{Ar}, \mathrm{Kr}$ and $\mathrm{Xe}$ clusters are given in figure 2 .

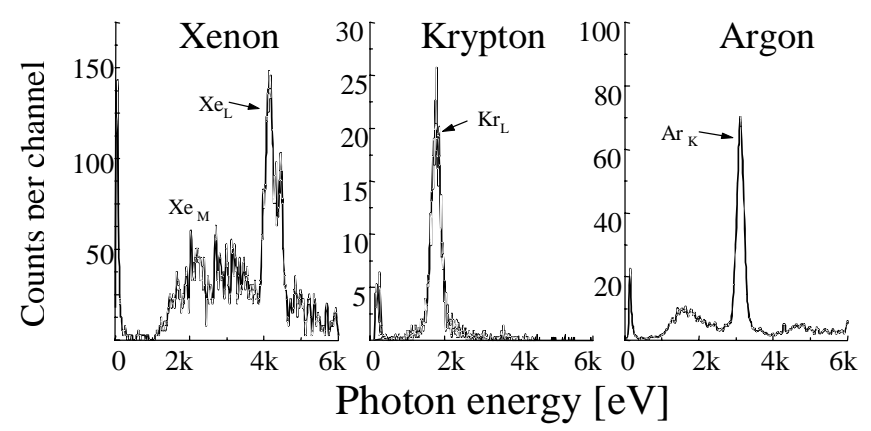

Fig. 2. One-photon spectra recorded during irradiation of argon, krypton and xenon clusters under similar conditions (130-fs infrared pulses at 790nm with a peak intensity of $5 \mathrm{x}$ $10^{17} \mathrm{~W} \mathrm{~cm}^{-2}$ and a backing pressure of $18 \mathrm{bar}$ ).

K-shell ionisation of argon and L-shell ionisation of krypton and xenon are observed. In each case, a study of the X-ray yield dependence on backing 
pressure and laser peak intensity has been performed. The results of this study are similar in each case ; a more extensive study has been done and more results obtained in the case of Ar highresolution spectroscopy, and the corresponding results will be given in the next subsection. We only give here quantitative results on the atomic species dependence, with measurements performed at the same (18 bar) backing pressure and laser peak intensity $\left(5 \times 10^{17} \mathrm{w} \mathrm{cm}^{-2}\right)$. Results are summarised in Table 1.

\begin{tabular}{|l|c|c|c|}
\hline & argon & krypton & xenon \\
\hline $\mathrm{E}_{\mathrm{h} v}(\mathrm{eV})$ & 3086 & $1741 / 2248$ & 4109 \\
\hline $\mathrm{N}_{\mathrm{hv}} /$ pulse & $7 \times 10^{4}$ & $4 \times 10^{6}$ & $1.5 \times 10^{5}$ \\
\hline$\langle\mathrm{Q}\rangle$ & 14 & $24-27$ & $31-35$ \\
\hline
\end{tabular}

Table 1. Energies, photon yields (total photon number per laser pulse) and mean charge states extracted in the case of argon, krypton and xenon clusters for the same (18 bar) backing pressure and laser peak intensity of $5 \times 10^{17} \mathrm{~W} \mathrm{~cm}^{-2}$.

The highest yield is obtained with krypton clusters. The observed low-resolution spectrum is in good concordance with the high-resolution spectrum observed by McPherson et al. [6]. With the geometry and the backing pressure used here, the atomic density in the interaction region is only $9 \mathrm{x}$ $10^{14} \mathrm{~cm}^{-3}$, whereas densities 5 orders of magnitude larger can easily be obtained by working at higher pressure and closer to the gas jet nozzle. For such densities, the photon yield would be in excess of $10^{11} \sim 2 \mathrm{keV}$ photon / pulse! The lowest yield corresponds to the argon case, and xenon is in between. At this stage, it is already worth noting that the observed mean charge states (deduced from Xray energies $[15,16])$ are much larger than the ones corresponding to direct observation of atomic debris from the cluster [2-4]. For instance, Lezius et al [3] have observed maximum charge states corresponding to $\mathrm{Ar}^{10+}$ and $\mathrm{Xe}^{30+}$ in similar conditions. These highly charged ions are in fact detected well after the early inner-shell ionisation stage under examination here, and certainly after some recombination with surrounding electrons has occured.

\subsection{High resolution results}

Though the observed yields are not as large in the case of argon as with other cluster targets, the observed X-ray transitions have here a relatively simple character : it is expected that only $1 \mathrm{~s} 2 \mathrm{p} 2 \mathrm{I}^{\mathrm{n}} \rightarrow$
$1 \mathrm{~s}^{2} 2 \mathrm{l}^{\mathrm{n}}$ transitions must be present in a high resolution spectrum, thus easing its interpretation (we note also that no $\mathrm{K} \beta$ transitions are found in the $\mathrm{Si}(\mathrm{Li})$ spectrum in this case, meaning that no $\mathrm{M}$ shell electrons remains at the time of K-shell X-ray emission). This is indeed the case, as observed in the sample spectrum presented in figure 3 corresponding to near optimum conditions (backing pressure $\mathrm{P}_{0}=$ 30bar, laser pulses of 80fs duration with a peak intensity of $6.7 \times 10^{17} \mathrm{~W} \mathrm{~cm}^{-2}$ ). $1 \mathrm{~s} 2 \mathrm{p}^{2} \mathrm{I}^{\mathrm{n}} \rightarrow 1 \mathrm{~s}^{2} 2 \mathrm{l}^{\mathrm{n}}$ transitions with $0 \leq \mathrm{n} \leq 5$ corresponding to charge states $11+-16+$ are observed, with a prominent and well separated peak corresponding to the ${ }^{1} \mathrm{P}_{1}$ transition in heliumlike argon. Since no hydrogenlike transition was observed, the ${ }^{1} \mathrm{P}_{1}$ line is free of satellites; its width, however, is found to be $4.5 \mathrm{ev}$, whereas the instrumental one is $1.5 \mathrm{ev}$ only. Hence, its width is due to physical processes (such as Stark Broadening for instance), and may be used to diagnose the cluster-scale nanoplasma at the time of X-ray emission.

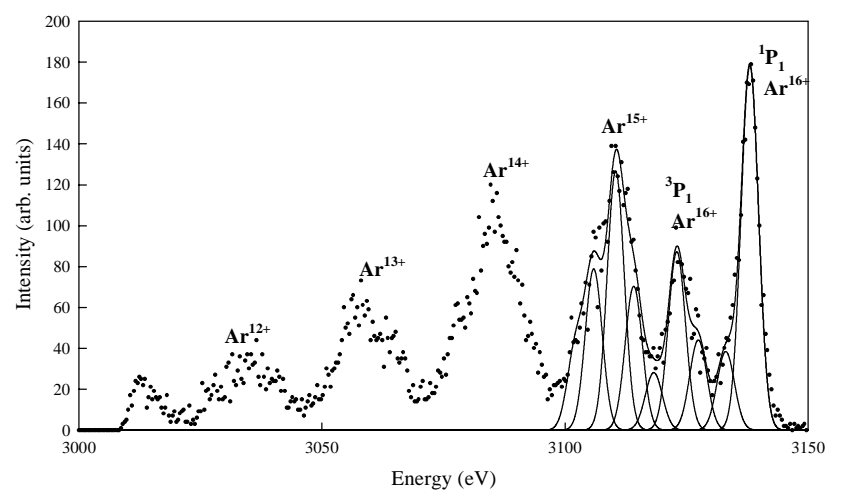

Fig. 3. High-resolution spectrum recorded after irradiation of argon clusters by $80 \mathrm{fs}$ infrared pulses at $790 \mathrm{~nm}$ with a peak intensity of $6.7 \times 10^{17} \mathrm{~W} \mathrm{~cm}^{-2}$ and a backing pressure of $30 \mathrm{bar}$.

Several spectra similar to this one have been recorded for various backing pressures and laser peak intensities. In each case, we have deduced the total X-ray yields (photon number per pulse) from both the high resolution spectrum and the simultaneously recorded $\mathrm{Si}(\mathrm{Li})$ ones. The mean photon energy, reflecting the mean charge state of the K-ionised emitting ions, was also deduced with high accuracy from the high resolution spectrum. Results corresponding to the laser energy dependence and the backing pressure one are given respectively in figure 4 and 5 .

The laser energy dependence is well fitted by a power law where the photon yield, $\mathrm{N}_{\mathrm{hv}}$, varies with the laser energy, or equivalently, the laser peak intensity, $\mathrm{I}_{\text {peak }}$, as

$$
\mathrm{N}_{\mathrm{hv}} \propto \mathrm{I}_{\text {peak }}^{3 / 2} .
$$


Such a behaviour has been observed previously in experiments concerning OFI of rare-gas atoms [14]. These studies have shown that, as soon as the ion yield of a given charge state enters the saturation regime, the signal increases further with $\mathrm{I}_{\text {peak }}{ }^{3 / 2}$. This behaviour is simply due to the increase with increasing $I_{\text {peak }}$ of the (focal) volume where a given threshold intensity is reached.
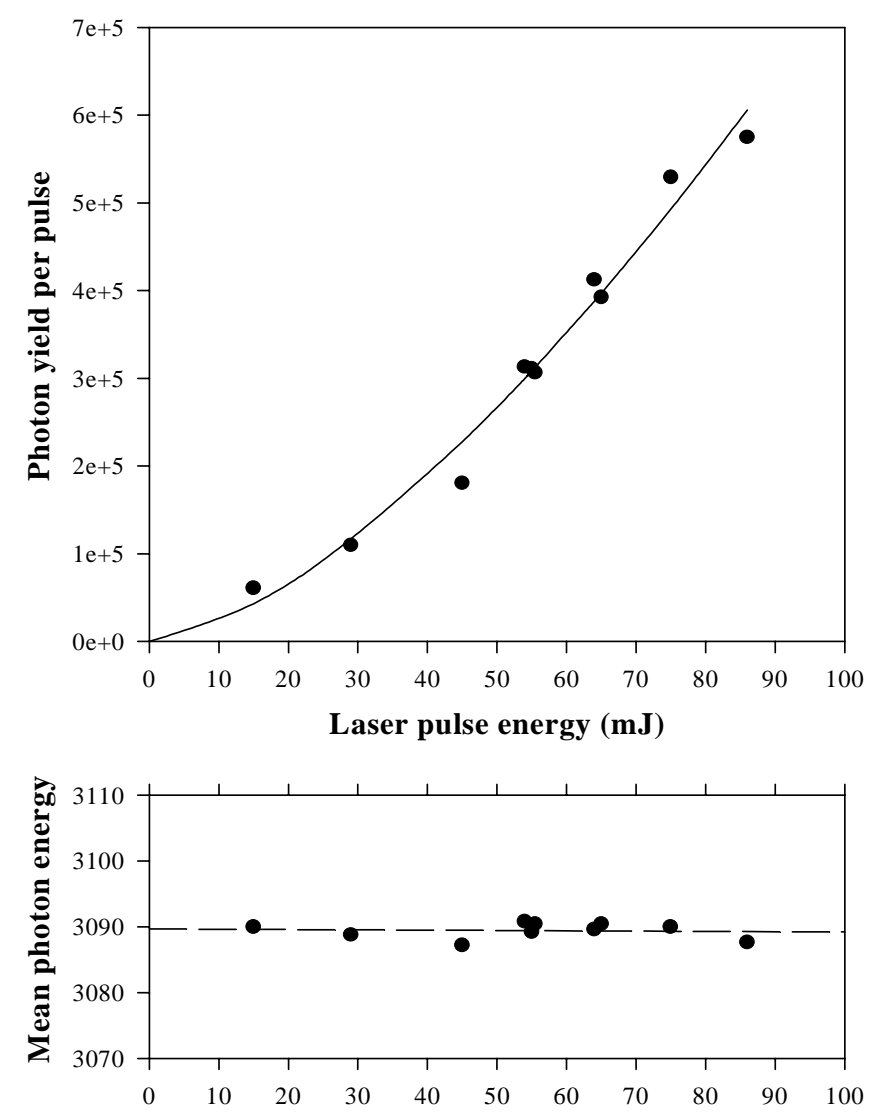

Figure 4. Argon cluster photon yield (upper curve) and mean photon energy (lower curve) dependence on the laser pulse energy recorded for $80 \mathrm{fs}$ pulses and a backing pressure of 26bar. The solid line in the upper curve is a fit with a power law of the form $\mathrm{E}_{\text {laser }}{ }^{3 / 2}$.

Our result indicate that the process of inner-shell ionisation is already saturated even at the lowest laser peak intensity used here $\left(\sim 10^{17} \mathrm{~W} \mathrm{~cm}^{-2}\right)$. It can then be expected that the onset of the X-ray generation should occur at even lower intensities. Note that in the case of krypton cluster, X-ray emission is similarly observed for laser peak intensities down to $\sim 4 \times 10^{16} \mathrm{~W} \mathrm{~cm}^{-2}$ [8]. The fact that the mean photon energy does not vary with the laser energy is also consistent with this picture : for a given cluster size, the ionisation probabilities in $\mathrm{K}$ and $\mathrm{L}$ shells are constant inside a given, laserintensity dependent, volume.

Whereas for a given backing pressure all spectra look the same, this is not the case when the cluster size is allowed to vary : a distinct dependence of the mean photon energy on the backing pressure is found here (fig. 5). Note that this energy variation has to be taken into account to extract the photon yields with the $\mathrm{Si}(\mathrm{Li})$ detectors, and much more accurate results are in fact obtained here using the high-resolution spectra. Analysis of the photon yield data indicate that the experimental result is best fitted with

$$
\mathrm{N}_{\mathrm{hv}} \propto\left(\mathrm{P}_{0}-\mathrm{P}_{\min }\right)^{5 / 3}
$$
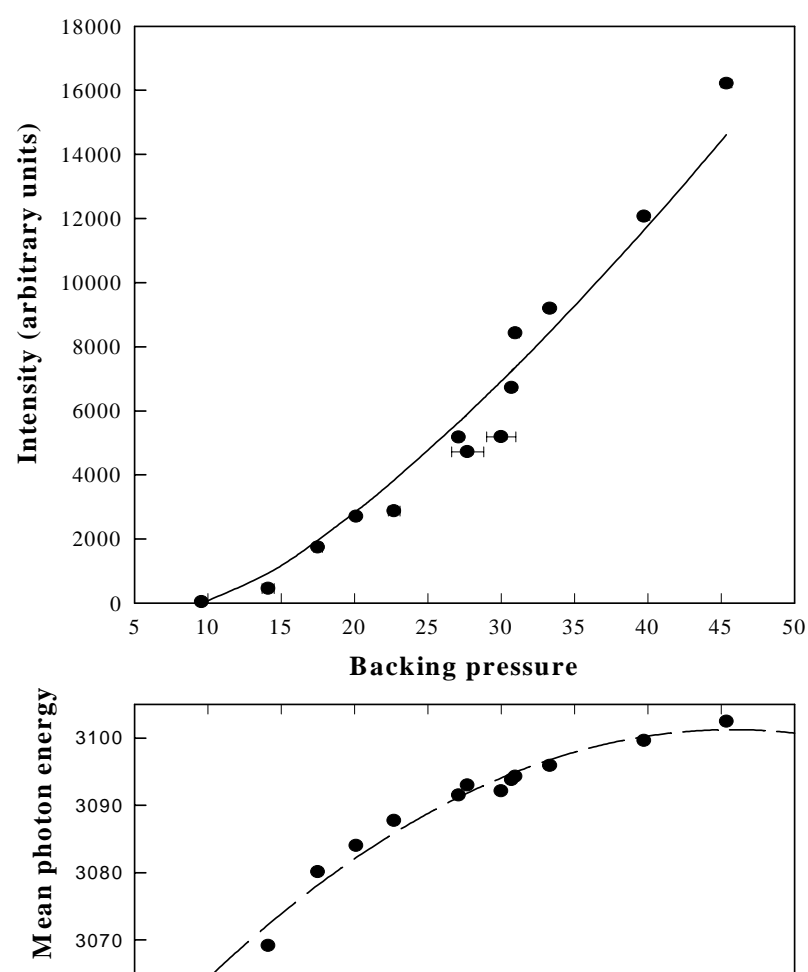

Figure 5. Argon cluster photon yield (upper curve) and mean photon energy (lower curve) dependence on the backing pressure recorded for $80 \mathrm{fs}$ pulses and a laser pulse energy of 90 $\mathrm{mJ}$. The solid line in the upper curve is a fit with a power law of the form $\mathrm{P}_{0}{ }^{5 / 3}$ where $\mathrm{P}_{0}$ is the backing pressure.

$\mathrm{P}_{\min }$ is found equal to $8 \mathrm{bar}$, corresponding to a cluster size of $\sim 10^{5}$ atoms/cluster. As will be shown now, equation (4) is consistent with a model in which X-ray emission occurs following electron impact ionisation inside each isolated cluster in the focal volume.

\section{Models and discussion}

At the present stage of our understanding of the physical processes involved in argon $\mathrm{K}$ and krypton or xenon L X-ray emission, we have found appropriate to distinguish, in our modelling, the inner-shell ionisation stage and the consecutive Xray emission. 


\subsection{Inner-shell ionisation model}

We first consider the case of argon clusters with the experimental conditions of table 1 (backing pressure $\mathrm{P}_{0}=18 \mathrm{bar}$ and 130fs laser pulses with a peak intensity $\mathrm{I}_{\text {peak }}=5 \times 10^{17} \mathrm{~W} \mathrm{~cm}^{-2}$ ). We assume the following scenario :

- at an early stage of the laser-cluster interaction, OFI of argon atoms occurs, producing $\mathrm{Ar}^{8+}$ ions and 8 quasi-free electrons. The threshold intensity for this process is estimated to be $2.6 \mathrm{x}$ $10^{16} \mathrm{~W} \mathrm{~cm}^{-2}$, whereas producing $\mathrm{Ar}^{9+}$ requires peak intensities exceeding $1.6 \times 10^{18} \mathrm{~W} \mathrm{~cm}-2$. Assuming a $\sin ^{2}$ time dependence for the laser intensity, $\mathrm{Ar}^{8+}$ ions are produced at least $110 \mathrm{fs}$ before the peak intensity

- later on, inner-shell ionisation results from ionisation and excitation processes induced by collisions between fast electrons and ionic species inside each isolated cluster. We consider that at this stage, only laser driven electrons may have sufficient energy to produce argon $\mathrm{K}$ shell vacancies. Taking a $4 \mathrm{keV}$ threshold for this process, a mean oscillation energy corresponding to this value is achieved for intensities of the order of $7 \times 10^{16} \mathrm{~W} \mathrm{~cm}^{-2}$, about $100 \mathrm{fs}$ before the peak intensity. From then on, the effective focal volume inside which this energy is achieved starts to increase, and reaches about 5 times the nominal focal volume at the peak intensity. In between, about 38 optical cycles have taken place.

- At the time when the peak intensity is reached, some increase in the cluster radius has already taken place. Numerical simulations [17] have shown that in the case considered here, the mean cluster radius has already increased by a factor of $\sim 3$, starts at that time to increase further very rapidly, and the collisional rate drops also very fast (see below). We then consider that no significant inner-shell ionisation can take place after that time.

Accordingly, the mean K-shell ionisation number of argon atoms per laser pulse is given by

$$
\mathrm{N}_{\mathrm{K}}=\mathrm{N}_{\text {cycle }} \mathrm{n}_{\mathrm{e}-\mathrm{n}} \sigma \times \mathrm{N}_{\mathrm{ag}},
$$

where $\mathrm{N}_{\text {cycle }}=38$ is the useful number of optical cycles, $\mathrm{n}_{\mathrm{e}}$ is the number of laser driven electrons in each cluster, $n=1 / r^{3}$ is the atomic density inside the cluster (and $r$ is the mean inter-atomic distance), $\sigma$ is the electron-induced $\mathrm{K}$-shell ionisation cross section, $x$ is the effective cluster thickness and $N_{a g}$ is the total number of clusters in the effective focal volume. We take $\mathrm{n}_{\mathrm{e}-}=8 \tilde{N}_{c}$ (8 "free" electrons per cluster atom) and, allowing for some cluster expansion, $r=2 r_{0}$ with $r_{0}=0.382 n m$ being the ground state mean inter-atomic distance in the cluster. The K-shell ionisation cross section [18] is in the range $1-3 \times 10^{-21} \mathrm{~cm}^{2}$ for electron energies 4$50 \mathrm{keV}$, and we take as a mean value $\sigma=2 \times 10^{-21}$ $\mathrm{cm}^{2}$. For a backing pressure $\mathrm{P}_{0}=18 \mathrm{bar}, \tilde{N}_{c}=5.9$ $10^{5}$ and the initial cluster radius is equal to $\sim 20 \mathrm{~nm}$. At laser intensities above $7 \times 10^{16} \mathrm{~W} \mathrm{~cm}^{-2}$, the amplitude of the electron oscillations in the laser field is $22 \mathrm{~nm}$, a value very close to the cluster radius. We take $\mathrm{x}=40 \mathrm{~nm}$ (the expanded cluster radius) as an order of magnitude. Finally, to evaluate the number of clusters in the effective focal volume, we take its time-averaged value which amounts to $\sim 2 \mathrm{~V}_{\text {foc }}$. The nominal focal volume, deduced from our laser intensity measurements, is $\mathrm{V}_{\text {foc }}=1.6 \times 10^{-8} \mathrm{~cm}^{3}$. With $\rho_{\mathrm{c}}=9 \times 10^{14} \mathrm{~cm}^{-3}$, we get $\mathrm{N}_{\mathrm{ag}} \sim 50$.

This gives $\mathrm{N}_{\mathrm{K}} \sim 1.6 \times 10^{5}$. Assuming finally a mean fluorescence yield $\widetilde{\omega}_{K}=0.35$ corresponding to the observed mean charge state [15], we predict an absolute photon yield/pulse of $6 \times 10^{4}$, in extremely good agreement with our experimental value of $7 \mathrm{x}$ $10^{4}$ (table 1$)$.

\subsection{Scaling laws : krypton and xenon clusters}

Equation (5) leads to the following scaling law : using $\mathrm{n}_{\mathrm{e}-}=\mathrm{n}_{\mathrm{OFI}} \tilde{N}_{c} \approx \mathrm{n}_{\mathrm{OFI}} \mathrm{A}_{\mathrm{c}} \mathrm{P}_{0}^{2}, \mathrm{n} \propto \mathrm{r}^{-3}, \mathrm{x} \propto$ $\left(\mathrm{A}_{\mathrm{c}} \tilde{N}_{c}\right)^{1 / 3} \mathrm{r} \propto \mathrm{A}_{\mathrm{c}}{ }^{1 / 3} \mathrm{P}_{0}{ }^{2 / 3} \mathrm{r}$ and $\mathrm{N}_{\mathrm{ag}} \propto \rho_{\mathrm{c}} / \tilde{N}_{c} \propto\left(\mathrm{A}_{\mathrm{c}}\right.$ $\left.\mathrm{P}_{0}\right)^{-1}$, one gets

$$
\mathrm{N}_{\mathrm{K}} \propto \mathrm{n}_{\mathrm{OFI}} \sigma \mathrm{A}_{\mathrm{c}}^{1 / 3} \mathrm{P}_{0}^{5 / 3} \mathrm{r}^{-2} \mathrm{~V}_{\text {foc }}\left(\mathrm{I}_{\mathrm{s}}\right),
$$

where $A_{c}$ is defined in equation (1), $n_{\mathrm{OFI}}$ is the number of electrons initially released by OFI, and $\mathrm{V}_{\text {foc }}\left(\mathrm{I}_{\mathrm{s}}\right)$ is the effective focal volume depending on the appropriate threshold electron energies.

First, one retrieves within this model the observed $\mathrm{P}_{0}{ }^{5 / 3}$ dependence.

The atomic species dependence is contained in $\mathrm{n}_{\mathrm{OFI}}$, $\sigma, A_{c}, r, V_{\text {foc }}\left(I_{s}\right)$ and in the effective fluorescence yields when converting to photon yields. Estimated values of theses quantities and predicted yields are given in table 2 . In our estimates, we have kept the krypton and xenon inter-atomic distances to their normal-cluster value, since cluster expansion is expected to occur later in these cases [17]. Also, we 
have used here the same effective fluorescence yield because calculations of this quantity is much harder to perform for L-shell transitions. This lead to an additional uncertainty in our estimates of a factor of about 2. Comparison of the results of our calculations in table 2 with the experimental yields of table 1 shows nevertheless that the agreement is more than qualitative.

\begin{tabular}{|l|c|c|c|}
\hline & Argon & krypton & xenon \\
\hline $\mathrm{n}_{\text {OFI }}$ & 8 & 8 & 12 \\
\hline$\sigma\left(10^{-21} \mathrm{~cm}^{2}\right)$ & 2 & 8 & 2 \\
\hline $\mathrm{A}_{\mathrm{c}}\left(\mathrm{x} \mathrm{10^{3 }}\right)$ & 2.1 & 6.7 & 23 \\
\hline $\mathrm{r}(\mathrm{nm})$ & 0.764 & 0.412 & 0.456 \\
\hline $\mathrm{V}_{\text {foc }}\left(\mathrm{I}_{\mathrm{s}}\right)\left(\mathrm{cm}^{2}\right)$ & $3.2 \times 10^{-8}$ & $7.4 \times 10^{-8}$ & $2.2 \times 10^{-8}$ \\
\hline $\mathrm{N}_{\mathrm{h} v} /$ pulse & $6 \times 10^{4}$ & $3 \times 10^{6}$ & $4 \times 10^{5}$ \\
\hline
\end{tabular}

Table 2. OFI released electron numbers, ionisation cross sections, scaling parameters for cluster size, mean inter-atomic distances in cluster, effective focal volumes and predicted photon yields (total photon number per laser pulse) for argon, krypton and xenon clusters for the same (18 bar) backing pressure and laser peak intensity of $5 \times 10^{17} \mathrm{~W} \mathrm{~cm}^{-2}$.

\subsection{Radiative decay and spectrum modelisation}

We now go back to the case of argon clusters and to the recorded high-resolution spectrum shown in figure 3. Our model predicts that near the maximum laser intensity, almost all $\mathrm{K}$ shell ionisation has already occurred. Electron mean energies ("temperature") may nevertheless be already large enough [1] to induce additional L shell ionisation, and certainly large enough to induce collisional mixing between various ionic configurations. However, 110fs later, the laser intensity falls below the $2.6 \times 10^{16} \mathrm{~W} \mathrm{~cm}^{-2}$ threshold and surrounding electrons may recombine in the $\mathrm{M}$ shell, and induce $\mathrm{K} \beta$ transitions if $\mathrm{K}$-vacancies are still present. Since we do not see such transitions, K-shell filling, by processes including $\mathrm{K} \mathrm{X}$-ray emission, must be almost complete at that time. We have added to figure 3 a tentative fit of the high-energy region of our spectrum by including only transitions from $1 \mathrm{~s} 2 \mathrm{p} 2 \mathrm{l}^{\mathrm{n}}$ states with lifetimes not exceeding the laser pulse duration. Such a fit reflects well the observation.

More sophisticated models can be used. We have used the HULLAC [19] code as a data base for the COLRAD [20] code. A stationary thermal distribution of electrons has been assumed. The electronic temperature $T_{e}$ and the electronic density $n_{e}$ have been adjusted to get the best fit. The result of our calculation gives satisfactory agreement with experiment (figure 6) for $\mathrm{T}_{\mathrm{e}}=750 \mathrm{eV}$ and $\mathrm{n}_{\mathrm{e}}=10^{21}$ $\mathrm{cm}^{-3}$. The extracted value for the electron temperature is much lower than the mean electron oscillation energy, and too small to lead to sizeable $\mathrm{K}$-shell ionisation. Assuming again 8 free electrons per cluster atom, the electronic density corresponds to an increase in the cluster radius of a factor of 5 . This is again an indication that X-ray emission must take place within at most few hundreds femtoseconds.

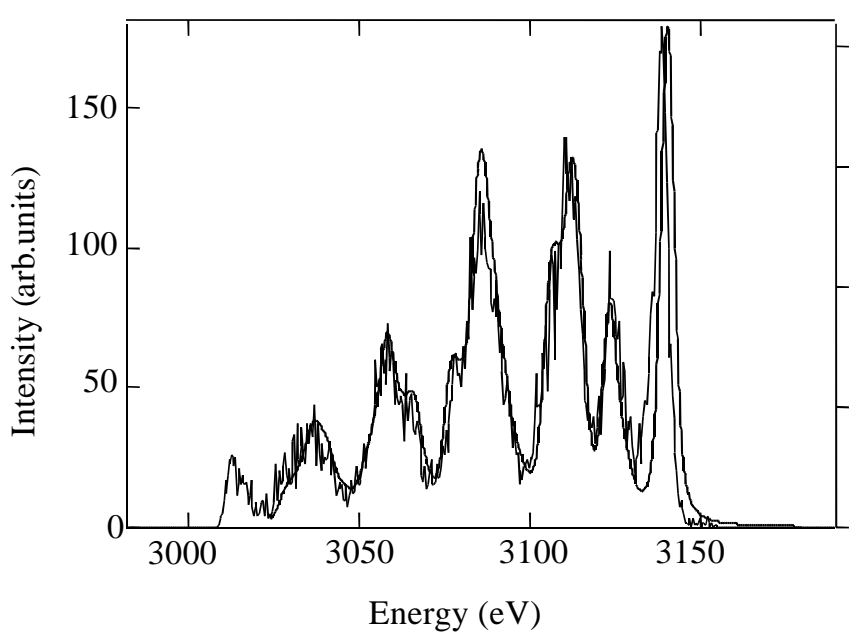

Figure 6. Comparison of the high-resolution argon spectrum with the COLRAD simulation (thin smooth line).

New numerical simulations including the inner-shell ionisation stage are in progress.

\section{Conclusion}

$\mathrm{KeV} \mathrm{X}$-ray production from irradiation of large rare-gas clusters by intense ultrashort laser pulses has been quantitatively studied. Variation laws with laser intensity, cluster size and atomic species have been obtained. A simple model taking into account the inner-shell ionisation induced by the laserdriven electron motion reproduces well the observed dependence and the absolute photon yields. However, it would be interesting to explore in more details the threshold behaviour embedded in our model, and to test laser wavelength, pulse duration and polarisation effects. The simulation of a recorded high-resolution spectrum indicate an electron temperature of $750 \mathrm{eV}$ and an electronic density of $10^{21} \mathrm{~cm}^{-3}$ at the time of X-ray emission. Our results and their interpretation suggest that $\mathrm{X}$ ray emission occurs on a very short time scale (few hundreds fs). The production of subpicosecond 
pulses of $10^{11}$ photons per laser pulse in the $\mathrm{keV}$ range seems to be at hand, and numerous applications may be foreseen.

\section{References :}

[1] Purnell, J. et al. ,1994, Chem. Phys. Lett. 229, 333

[2] Snyder, E. M. et al. ,1996, Phys. Rev. Lett. 77, $\mathrm{xxx}$

[3] Lezius, M. et al., 1997, J. Phys. B : At. Mol. Opt. Phys. 30 L251

[4] Ditmire, T. et al., 1997, Nature 38654

[5] Wülker, C. et al., 1994, Opt. Commun. 112, 21

[6] McPherson, A. et al., 1994, Phys. Rev. Lett. 72, 1810

[7] Ditmire, T. et a.l, 1995, Phys. Rev Lett. 75, 3122; Ditmire, T. et al., 1996, Phys. Rev. A $\mathbf{5 3}, 3379$

[8] Dobosz, S. et al., 1997, Phys.Rev. A 56, 4, 1

[9] Lezius, M. et al., 1997, OSA VII ${ }^{\text {th }}$ proceeding, Santa Fe (USA)

[10] Kondo, K. et al., 1998, J. Phys. B : At. Mol. Opt. Phys. 302707

[11] Ditmire, T. et al., 1998, J. Phys. B : At. Mol. Opt. Phys. 312825

[12] Vernhet, D. et al., 1998, J. Phys. B : At. Mol. Opt. Phys. 313949

[13] Hagena, O. F. and Obert W., 1972, J. Chem. Phys. 56, 1793

[14] Augst, S. et al, 1989, Phys. Rev. Lett. 63, 2212

[15] Bhalla, C. P., 1973, Phys. Rev. A 8, 2877

[16] Cornille, M., Private communication.

[17] Dobosz, S., 1998, These de doctorat Université Paris 13.

[18] Hippler, R. and Jitschin, W., 1982, Z. Phys. A 307, 287

[19] Bar-Shalom, A., Klapisch, M. and Goldstein, W. H., 1998, the HULLAC code for atomic physics, (unpublished)

[20]Ljepojevi, N. N. , Hutcheon, R. J. and Payne, J., 1987,Comput. Phys. Commun. 44, 157 